\title{
New advances in airflow measurements applied in airflow characteristics test of pneumatic components
}

\author{
SHI Yan ${ }^{1}$, HAN ChengWei ${ }^{1}$, CAI MaoLin ${ }^{1}$, WANG YiXuan ${ }^{1} \&$ SUN ZhiBo ${ }^{2 *}$ \\ ${ }^{1}$ School of Automation Science and Electrical Engineering, Beihang University, Beijing 100191, China; \\ ${ }^{2}$ Engineering Training Centre, Beihang University, Beijing 100191, China
}

Received May 18, 2021; accepted October 13, 2021; published online November 4, 2021 Citation: Shi Y, Han C W, Cai M L, et al. New advances in airflow measurements applied in airflow characteristics test of pneumatic components. Sci China
Tech Sci, 2021, 64: 2781-2782, https://doi.org/10.1007/s11431-021-1946-9

Pneumatic components have the explosion-proof, high reliability, and pollution-free characteristics, and have been applied in high-end equipment such as aerospace. With the pneumatic system technology developing, the quality of pneumatic components is required improving [1]. High-frequency airflow dynamic characteristics need to be applied in pneumatic components. The quality and quantity analyses of gas flow components can provide significant information for estimating. However, there is not reliable method to determine the flow-rate [2]. This article is going to talk about the gas flow measurements which can be applied in airflow characteristics test.

Pan et al. [3] and Xu et al. [4] proposed a method which is least squares support vector machines (LS-SVM) based on temperature time series for gas flow measurement. This method used the non-invasive technique that has sensor installed outside the pipeline. In other word, the sensor is not directly connected with gas. The result of the experiment showed that the time spend on signal collecting can be effectively decreased without negative influence on the result. In the contrast, the accuracy of this method was still low compare to the other invasive flowmeters. However, the range of estimations and installation guidance can be guarantee by this method for permanent flowmeters [3].

Sun et al. [5] and Fan et al. [6] proposed a non-intrusive and non-invasive method to do the measurement of gas flow rates. The result of the experiments showed that the mea-

*Corresponding author (email: sunzb@buaa.edu.cn) surement method can illustrate the flow rate continuously, but need to enhance the accuracy of the measurements.

In 2020, a method was mentioned that can obtain the frequency data of pneumatic system, which was talk about by Al-Alweet et al. [7]. The significance of the relation between frequency and statistical parameters was given. By corresponding the capacitance values and the interrelation between frequency and statistical parameters, difference of flow patterns can be obtained.

The experiment of a thermal mass flow meter (TMFM) which was aiming to perform the steady-state heat transfer phenomena in the sensors was done by Kim et al. [8] in 2007. The result of the experiment demonstrated that the simple numerical model was validated. Moreover, the predicting correlation of the TMFM linear range was achieved with the $15 \%$ relative error.

In 2021, Zhang et al. [9] used the computational fluid dynamics digital elevation model (CFD-DEM) coupling simulation method to achieve the comparation of the characteristics of gas-solid flow. According to the simulation results, the conclusions below can be obtained.

(1) With the rotation drill pipe speed increasing, the distance and velocity of the gas flow increase.

(2) Because of the drill pipe rotation, the mass flow rate of the particle and the drill pipe solid-gas ratio were less than ideal.

For the CFD-DEM method running processes are showing in Figure 1.

In 2020, Li et al. [10] using adaptive combining com- 


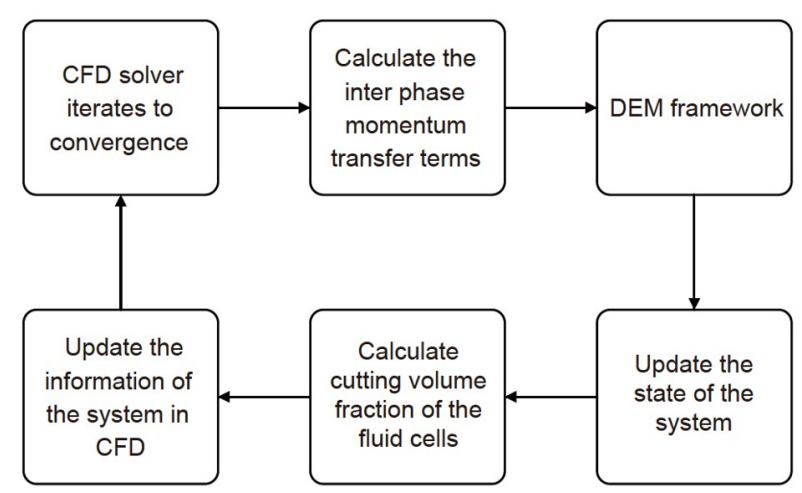

Figure 1 the CFD-DEM coupling process [7].

plementary ensemble empirical mode decomposition-artificial neural network (CEEMD-ANN) algorithm in pneumatic flow pattern identification. The results showed that the accuracy can be guarantee in this way. Moreover, the algorithm can avoid useless calculation in performing the features of pneumatic flow pattern, which means the real-time performance of the pneumatic flow was enhanced.

In conclusion, there are many methods to obtain the airflow characteristics. The method LS-SVM can provide wide range of gas flow temperature measures. The method based on TMFM achieved $15 \%$ relative error in performing steadystate heat transfer in sensors. Moreover, CFD-DEM can provide a comparation to estimate the gas flow. All the methods mentioned above have their advantages and disadvantages. The combination of TMFM and LS-SVM can increase the accuracy in temperature testing of LS-SVM. The CFD-DEM coupling simulation can achieve the comparation of the gas-solid flow, which means the comparation can provide important information of gas flow for LS-SVM and
TMFM combination. Moreover, CEEMD-ANN algorithm can be used for avoid useless calculation. In the further study, the effective combination of these methods should be made to achieve high-efficiency gas flow characteristics measurements for pneumatic components.

This work was supported by the National Natural Science Foundation of China (Grant No. 51975024) and the China Postdoctoral Science Foundation (Grant No. 2019M660392).

1 Shi Y, Wu T, Cai M, et al. Energy conversion characteristics of a hydropneumatic transformer in a sustainable-energy vehicle. Appl Energy, 2016, 171: 77-85

2 Kim S H, Lee G. An analytical method for gas flow measurement using conservative chemical elements. Appl Sci, 2021, 11: 6415

3 Pan H, Sun W, Sun Q, et al. Deep learning based data fusion for sensor fault diagnosis and tolerance in autonomous vehicles. Chin J Mech Eng, 2021, 34: 72

4 Xu W, Fan Z, Cai M, et al. Soft sensing method of LS-SVM using temperature time series for gas flow measurements. Metrol Meas Syst, 2015, 22: 383-392

5 Sun X Q, Wang Y J, Cai Y F, et al. An adaptive nonsingular fast terminal sliding mode control for yaw stability control of bus based on sti tire model. Chin J Mech Eng, 2021, doi: 10.21203/rs.3.rs-36541/v2

6 Fan Z C, Cai M L, Xu W Q. Non-invasive and non-intrusive gas flow measurement based on the dynamic thermal characteristics of a pipeline. Meas Sci Technol, 2012, 23: 105303

7 Al-Alweet F M, Jaworski A J, Alghamdi Y A, et al. Systematic frequency and statistical analysis approach to identify different gas-liquid flow patterns using two electrodes capacitance sensor: experimental evaluations. Energies, 2020, 13: 2932

8 Kim D K, Han I Y, Kim S J. Study on the steady-state characteristics of the sensor tube of a thermal mass flow meter. Int $\mathrm{J}$ Heat Mass Transfer, 2006, 50: 1206-1211

9 Zhang H, Zhang O, Li B, et al. Effect of drill pipe rotation on gas-solid flow characteristics of negative pressure pneumatic conveying using CFD-DEM simulation. Powder Tech, 2021, 387: 48-60

10 Li J, He K, Tan M, et al. An adaptive CEEMD-ANN algorithm and its application in pneumatic conveying flow pattern identification. Flow Meas Instrum, 2021, 77: 101860 